\title{
Thermochemical modelling of the subsurface environment on Enceladus
}

\author{
R. E. HAMP*, K. OLSSON-FRANCIS, S. P. SCHWENZER \\ AND V. K. PEARSON \\ Faculty of Science, Technology, Engineering and \\ Mathematics, The Open Univerisy, Milton Keynes \\ (*rachael.hamp@open.ac.uk)
}

The subsurface environment of Enceladus is potentially habitable: there is a global subsurface ocean [1], energy from hydrothermal activity [2] and bioesential elements [3]. Carbon, as a fundamental bioessential element, it is critical for life, so understanding how it is processed within the Enceldus environment is crucial in assessing Enceladus' potential habitability. Carbon is likely to be bound within the silicate interior [4] and liberated through water-rock (silicateocean) interactions.

We have undertaken thermochemical modelling (CHIMXPT) [5] of these interactions and tested different hypotheses for the formation of Enceladus. Both models reacted the silicate interior (with a CI chondrite compositon [6]) with a fluid representative of the subsurface ocean: a) a dilute sodium chloride solution, based upon the assumption that the subsurface ocean originated as almost pure water [7]; b) a solution with a cometary composition based upon data collected from 67P [8], based upon the assumption that the water originated from melted cometary ice [9]. We have explored the full temperature and pressure ranges anticipated at the rock-water interface [2].

We will present the outcomes from this modelling, which includes a theoretical compostion for a modern day subsurface ocean, potential carbon cycling pathways and the effect of carbon species on the $\mathrm{pH}$ of the subsurface ocean fluid.

[1] Thomas P. C. et al., (2016), Icarus, 264, 37-47 [2] Hsu H. W. et al., (2015), Nature, 519, 207-210 [3] McKay, C. P. et al., (2014) Astro-biology, 14, 352-355 [4] Glein C. R. \& Waite J. H., (2020), Geophys Res Let, 47 [5] Reed, M. H., Spycher, N. F., Palandri, J., (2010) User guide for CHIM-XPT, University of Oregon, Oregon [6] Hamp R. E. et al., (2019), 50th LPSC 2019, Abstract 1091 [7] Brown R. H. et al, (2006) Science, 311, 1425-1428 [8] Hertier K. H., et al., (2017) RAS monthly notices, 469 [9] Neveu, M., et al., (2017) Geochim et Cosmochim, 212, 324-371 